Parent descriptions of the presentation and management of anxiousness in children on the autism spectrum

Dawn Adams ${ }^{1,2,3}$, Kate Young ${ }^{1}$, Kate Simpson ${ }^{1,2,3}$ and Deb Keen ${ }^{1,2,3}$

${ }^{1}$ Autism Centre of Excellence, School of Education and Professional Studies, Griffith University, Brisbane, Australia

${ }^{2}$ Griffith Institute of Educational Research, Griffith University, Brisbane, Australia

${ }^{3}$ Cooperative Research Centre for Living with Autism (Autism CRC), Australia

Running Head: Parent descriptors of anxiousness in autism

Conflict of Interest: The authors declare that they have no conflict of interest.

Correspondence regarding this article should be addressed to:

Dr Dawn Adams

Autism Centre of Excellence

School of Education and Professional Studies

Griffith University

Mount Gravatt, Brisbane

QLD 4122

Australia

Email: Dawn.Adams@Griffith.edu.au 


\section{Acknowledgements}

We are grateful to the children with autism and their families for giving their time to support this research study. The authors acknowledge the work of the LASA team members: Jacqui Roberts (Project Leader), Susan Bruck, Trevor Clark, Sandra Devaraj, Robyn Garland, Honey Heussler, Antoinette Hodge, Patricia Howlin, Jessica Paynter, Natalie Silove, David Trembath, Madonna Tucker, Marleen Westerveld, Katrina Williams.

\section{Funding statement and declaration of conflicting interests}

The authors have no conflict of interest with respect to this publication.

The authors acknowledge the financial support of the Cooperative Research Centre for Living with Autism (Autism CRC), established and supported under the Australian Government's Cooperative Research Centres Program. The views expressed are those of the author(s) and not necessarily those of any of the funding bodies. 


\title{
Parent descriptions of the presentation and management of anxiousness in children on the autism spectrum
}

\begin{abstract}
Background: The complex interaction between anxiety and autism has led to debate about the presentation of anxiety in individuals on the spectrum and questions about the extent to which traditional checklists assess the entire range of symptomatology. Moreover, studies to date have not explored how the presentation of anxiety may differ between settings.

Methods: Through a combination of open-ended questions, closed questions and standardised measures, parents of 173 children (aged 6-13) on the autism spectrum provided (1) descriptors of their child's anxiety at home, school, and in the community and (2) strategies used to reduce their child's anxiety in each setting. Results: Over half (52.6\%) felt their child was anxious at home, $77.6 \%$ at school, and $76.2 \%$ in the community. Parents reported differing presentations of anxiety between settings, with the majority of descriptions relating to observable, behavioural changes (e.g., hides/shuts down, repetitive behaviours) rather than cognitive or physiological signs. Parents also reported using different strategies across settings.

Conclusions: The use of open-ended questions allowed the identification of signs of anxiety not explored within traditional questionnaires and highlighted the potential for signs to vary across settings. This knowledge is critical to inform the development or adaptations of anxiety measures and interventions.
\end{abstract}

Keywords:

autism, anxiety, mental health, parent report, qualitative 


\section{Parent descriptions of the presentation and management of anxiousness in children on the autism spectrum}

Anxiety is recognised as one of the most common co-occurring diagnoses for children on the autism spectrum, with meta-analyses placing the prevalence at around $40 \%$ (van Steensel, Bögels, \& Perrin, 2011) compared to $13.4 \%$ of children worldwide without autism (Polanczyk, Salum, Sugaya, Caye, \& Rohde, 2015). In addition to this $40 \%$, many individuals on the autism spectrum experience anxiousness or "sub-clinical" levels of anxiety (Strang et al., 2012; Vasa et al., 2013).

Anxiety is a complex and multi-faceted disorder which involves behaviours, cognitions, affect, and physiological arousal (Beidel, Turner, \& Dancu, 1985), some aspects of which may overlap with autism characteristics (White, Oswald, Ollendick, \& Scahill, 2009). The complex interactions between anxiety symptoms and autism characteristics have given rise to some uncertainty about the presentation of anxiety in individuals on the spectrum. Earlier research (Lecavalier, Gadow, DeVincent, \& Edwards, 2009; Weisbrot, Gadow, DeVincent, \& Pomeroy, 2005) indicated that the pattern and intensity of anxiety symptoms found in children on the autism spectrum were similar to symptoms identified in typically developing children. However, other authors (e.g. Kerns \& Kendall, 2012) have suggested that individuals on the autism spectrum may experience both traditional and atypical anxiety. Examples of atypical anxiety noted in individuals on the autism spectrum include anxiety around routine, novelty and restricted interests, unusual specific fears, social fearfulness and compulsive/ritualistic behaviour (see Kerns et al., 2014, for examples from within their sample). These are in contrast to the traditional, DSM-consistent anxiety, for example, 
common phobias (e.g. dogs, snakes), social phobia (e.g. an undue fear of social ridicule or rejection in the presence of social motivation) or obsessive-compulsive behaviours that can be clearly connected to a need to prevent or mitigate distress or a dreaded situation occurring.

This ongoing discussion within the literature as to the presentation of anxiety within individuals on the autism spectrum is further reflected in the debate around the appropriateness of using anxiety measures designed and validated on the general population to assess anxiety in autism. A working group empanelled by Autism Speaks concluded that these measures were not entirely appropriate for use in children on the autism spectrum (Lecavalier et al., 2014). Other researchers have argued for the utility of such measures even though they yield different factor structures in autistic and nonautistic populations (e.g. Magiati, Lerh, et al., 2017). After a critical review of the literature in this area, Magiati, Ozsivadijan, and Kerns (2017) concluded that whilst existing anxiety measures developed for typically developing children are useful to some extent, they are not likely to capture the full range of anxiety presentations seen in individuals on the autism spectrum and that work needs to go beyond reporting total and subscale scores if our understanding of anxiety in autism is to be enriched.

Existing standardised anxiety measures may thus have limitations when it comes to describing the profile of anxiety symptoms in individuals on the autism spectrum, and other methodologies may be needed to enhance our knowledge and understanding of anxiety in autism. To this end, some researchers have explored the modification or development of anxiety measures that take account of atypical symptoms of anxiety. Kerns et al. (2014) used an expanded version of the Anxiety Disorders Interview Schedule: Child and Parent versions and reported the presence of traditional (17\%), atypical (15\%), or both traditional and atypical (31\%) anxiety symptoms in their 
sample. Rodgers et al. (2016) developed an autism-specific measure of anxiety, the Anxiety Scale for Children - Autism Spectrum Disorder (ASC-ASD). While further validation of this measure is needed, initial results from the test developers and independent research groups suggest good psychometric properties, including good internal consistency ( $\alpha>$.8; Keen, Adams, Simpson, den Houting, \& Roberts, 2017) and validity (e.g. discriminant validity; den Houting, Adams, Roberts, \& Keen, 2018). Findings from this research have led to a call for more standardised, comprehensive, and multidimensional characterisation of atypical anxiety in autism.

In addition to the use of standardised measures, qualitative approaches have the potential to further our understanding of anxiety symptomatology in autism, but to date have received little attention in the research literature. Ozsivadijan, Knott, and Magiati (2012) reported on qualitative data gathered from focus groups with 17 caregivers of children on the autism spectrum aged 7-18 years. Parents described specific behavioural, somatic, and cognitive presentations of anxiety, which Ozsivadijan et al. grouped into challenging behaviour (including meltdowns, physical and verbal aggression), avoidance/withdrawal/escape, arousal (including increased levels of activity, nervous behaviour), sensory behaviour (including nail-biting and chewing of clothes), obsessional and repetitive behaviours, and somatic indicators (including sleep disruptions and nausea). There was also a strong theme around the difficulty children had in telling others they were anxious. The parents identified that their children on the autism spectrum tend to express anxiety behaviourally whereas they noted that typically developing children tended to express anxiety verbally.

Bearss et al. (2016) also noted the presence of observable behaviours (as opposed to verbal descriptions) to indicate anxiety when reporting on the qualitative analysis of focus groups with parents of 45 children on the autism spectrum aged 3-17 
years. Parents in the study reported children showing somatic responses, becoming overwhelmed and tearful, "acting out”, self-injurious behaviour (including skin-picking), sleep difficulties, self-deprecation, and facial expressions of fear, panic, or a blank expression. The behavioural manifestation of anxiety has also been reported in qualitative studies with adults on the autism spectrum (Robertson et al., 2018; Trembath, Germano, Johanson, \& Dissanayake, 2012).

The two qualitative studies discussed above make an important contribution to our understanding of how anxiety may present in children with autism; however, they involved small sample sizes (11-48 parents reporting on 11-45 children) drawn from specific geographical regions. Furthermore, reliance on focus group methodology may carry a sampling bias towards those willing and able to attend such a group. Exploration of parent descriptions of anxiety involving larger samples across broader geographical boundaries is therefore warranted.

Overall, the results from standardised measures and qualitative observational studies reviewed above support Magiati et al.'s (2017) conclusion that standardised questionnaires not specifically designed for children on the autism spectrum may fail to detect some of the observable, behavioural indicators of anxiety in individuals on the autism spectrum. There is thus a risk that anxiety may go unrecognised and therefore untreated. There have been numerous treatment trials for anxiety in individuals on the autism spectrum (e.g. Vasa et al., 2014), particularly cognitive-behavioural therapy (Spain, Sin, Harwood, Mendez, \& Happé, 2017; Ung, Selles, Small, \& Storch, 2015), showing positive outcomes. If anxiety is undetected, many parents may be left to manage their child's anxiety using non-manualised or ad-hoc strategies. Qualitative studies exploring the "lived experience" of how parents or individuals on the autism spectrum manage their anxiety identify a number of overlapping strategies. Bearss et al. 
(2016), from their focus groups of parents of 45 children on the autism spectrum, identified three themes relating to management: coping, parental management, and hold in-escalation-release. The coping theme described strategies the child employed when experiencing anxiety, including physical or mental withdrawal, comfort seeking (from a caregiver), repetitive behaviours, discussing the anxiety, seeking reassurance, imposing rules on others, and self-exertion (attempting to work off anxiety). Parental management included controlling the environment, giving details about upcoming events, giving the child freedom for action or thought, providing reassurance, and exposure/role playing. The hold in-escalation-release theme described the child trying to "hold in" the anxiety for a period of time, which then resulted in a build-up and a large release (often a temper outburst). The role of parents and caregivers' management and support is clearly important given that it continues to play a significant role in managing anxiety into adulthood (Robertson et al., 2018; Trembath et al., 2012). As discussed in Sze and Wood (2008) and Ozsivadijan et al. (2012), gaining further information on the strategies that families and children have found helpful to reduce anxiety across multiple settings may help to inform ASD-specific anxiety intervention development or ASD-specific modifications to existing interventions.

Given the recognition of the importance of using approaches that allow for open and non-directed responses to describe anxiety and anxiousness in individuals on the autism spectrum and to address the limitations of the previous qualitative studies, this study used a bottom-up approach (Ozsivadjian et al., 2012): instead of asking parents which predetermined symptoms of anxiety their child showed, we asked them to tell us what signs of anxiety or anxiousness they see in their child. The aim of this research was to document parental perspective of signs of anxiety or anxiousness in children on the autism spectrum and to document the strategies that they reported being helpful in 
reducing their child's level of anxiousness. The secondary aim was to explore whether these signs differed between home, school, and community settings, or with child age or gender. In order to meet these aims, the following research questions were asked:

1) What proportion of parents identifies their child as anxious at home, at school, or in the community and does this differ with child age or gender?

2) What signs of anxiousness do parents report at home, at school, or in the community?

3) Are these signs of anxiousness perceived as "typical” or autism-specific signs of anxiety?

4) What strategies do parents report being helpful to reduce their child's anxiousness?

\section{Methods}

This study used data collected as part of the Longitudinal study of Australian Students with Autism (LASA). Ethical approval for this study was obtained from all participating universities and health authorities.

\section{Recruitment Procedures}

The full recruitment procedure for the larger LASA cross-sequential study is described in Roberts et al. (2018). In brief, parents of children on the autism spectrum aged 4-5 or 9-10 years living in Australia were invited through clinics and advertisements on social media to take part in a 6-year study with annual data collection through an online survey. The sample was therefore self-selecting and for reasons of confidentiality and practicality, the research team was not provided with any information on families who may have been provided with recruitment information but who did not choose to enrol into the study. Upon entry into the study, all participants were requested to provide copies of their diagnostic reports as well as to complete a measure of autism characteristics (Social Communication Questionnaire, SCQ; Rutter, Bailey, Berument, Lord, \& Pickles, 2003). 
The data for this study were collected in the third year of data collection of the larger study, as this is the first year that the open-ended questions on anxiety were added into the survey.

\section{Participants}

All 272 parents who were recruited into the study are invited to complete the survey each year. A total of 174 parents provided responses for the questions relevant to this study. One child was excluded from this study as their SCQ score was below 15 and no community diagnostic report was available. To reflect the heterogeneity of a community sample, no exclusions were made based on the presence or absence of any additional psychiatric or neurodevelopmental diagnoses. There were no significant differences in responders and non-responders in gender $\left(\chi^{2}(1)=.22, p=.64\right)$, age at enrolment into the study $(\mathrm{t}(270)=1.04, \mathrm{p}=.30)$, parent education $(\mathrm{U}=8189, \mathrm{p}=.54)$, or family income $(U=7605, p=.14)$.

The final sample for this study consisted of data on 173 children on the autism spectrum, living in Australia. The sample consisted of 139 males (80.3\%) and 34 females (19.7\%). Due to the study design, at the time the questionnaire was completed the 88 children from the younger cohort were aged between 6 years 0 months and 8 years 11 months $(\overline{\bar{x}}=86.2$ months, $S D 10.0)$ and the 85 children from the older cohort were between 12 years 11 months and 13 years 5 months $(\overline{\bar{x}}=145.4$ months, $S D$ 7.5). Parents were asked if their children had a diagnosis of an anxiety disorder; 63 (36.4\%) parents reported that their child had a diagnosis of anxiety, 19 of whom were from the younger cohort and 44 from the older cohort. No information was gathered on anxiety diagnosis subtypes. In total, 27 (15.6\%) - 21 of whom were from the older cohort were reported to be prescribed selective serotonin reuptake inhibitors (SSRIs).

\section{Measures}


Autism characteristics. The SCQ (Rutter et al., 2003) is a behavioural checklist that requires parents to indicate the presence of certain social, communicative, or stereotyped behaviours by answering "yes" or "no" to 40 items. The SCQ has been extensively researched, with a recent meta-analysis (Chesnut, Wei, Barnard-Brak, \& Richman, 2017) concluding that it is an acceptable screening measure for autism spectrum disorder (area under the curve .89) if used within the correct age range. A higher score represents a higher number of behaviours which may be considered indicative of autism. Children who scored below the cut-off of 15 on this measure were only included if they had community diagnostic reports from a paediatrician and/or psychologist confirming an autism diagnosis.

Anxiety symptomatology. The Anxiety Scale for Children with Autism Spectrum Disorder Parent form (ASC-ASD-P; Rodgers et al., 2016) was available for 171 children in this sample. The ASC-ASD-P consists of 24 items from which four subscales have been derived: Performance Anxiety, Anxious Arousal, Separation Anxiety, and Uncertainty. Severity was rated on a 4-point scale ranging from 0 (never) to 3 (always). Although the scale was originally developed for children aged 8 or above, it has been used in younger samples of children on the autism spectrum to describe anxiety symptomatology (e.g. Keen et al., 2017). The scale has good validity, reliability, and internal consistency and is highly correlated with the Screen for Child Anxiety Related Emotional Disorders (SCARED; Birmaher et al., 1999), a robust measure of anxiety in the general population. In the current study, internal consistency was identified as acceptable to excellent for all subscales based on Cronbach's alpha; Anxious Arousal $\alpha=.87$, Separation Anxiety $\alpha=.83$, Performance Anxiety $\alpha=.89$, and Uncertainty $\alpha=.90$.

\section{Questions on signs of anxiety and anxiousness and helpful management}

strategies. Parents were asked a combination of closed-and open-answer questions 
regarding their child's anxiety profile across home, school, and community settings. Parents were firstly asked if they felt that their child was anxious at home. If they stated "yes", they were then asked two open-answer questions: (a) to describe their child's signs of anxiety at home, and (b) to state the strategies they have found helpful in reducing their child's anxiety at home. The questions were then repeated for school and then for the community. The questionnaire did not ask parents how they became aware of the signs of their child's anxiety at school or in community settings.

\section{Data Analysis}

The closed questions (whether parents feel their child is anxious at home, at school, or in the community) were analysed using SPSS. Group comparisons were conducted with $\chi 2$ and effect sizes reported using $w$ (where for 1, degree of freedom, .10 is a small effect size, .30 is a medium effect size, and .50 is a large effect size). To provide an assessment of concurrent validity for these questions, scores on the autism-specific measure of anxiety, the ASC-ASD (Rodgers et al., 2016), were compared between those who were and were not reported to be anxious across the three settings.

Signs of each child's anxiousness and helpful anxiety-reduction strategies reported by parents were open-ended answers. The responses were analysed using content analysis by one of the authors using the four steps described by Dey (1993) as used in previous autism research (e.g. Baron-Cohen \& Wheelwright, 1999; Johnson \& Hastings, 2002). The steps of Dey were (a) divide the data into manageable parts, (b) collect responses together that relate to the areas or questions of interest, (c) create categories that describe similar responses within these general groupings, and (d) combine or split categories where data can best be described in a rearranged structure. Once categories were created, these were discussed with the first author and clarity was added to category names or items. 
Reliability of the content analysis was assessed by having a second, independent person place $20 \%$ of the responses into categories. The extent of agreement between the two coders was calculated using a simple percentage agreement index formula $\{[$ agreements $/$ (agreements + disagreements $)] \times 100 \%\}$. An agreement was defined as when both raters coded a given answer into the same category. Initial inter-rater agreement was $60-70 \%$ across both questions (signs and strategies) in all contexts (home, school, community). After feedback discussions between the raters, clarifications were made to category names and labels, and the inter-rater process repeated. Overall final agreement for the signs and strategies was 95-100\%.

As category descriptors are marked as either being present or absent (regardless of the number of descriptors given), if parents provided a number of descriptors within one category (e.g., "shouts, gets louder"), it was only marked as "present".

In order to explore whether the signs of anxiousness reported by parents were perceived as "typical" (i.e. DSM-consistent) or autism-specific signs of anxiousness, the categories and examples in Table 2 were sent to psychologists and clinical psychologists working in the area of autism and/or anxiety. They were asked to rate whether they perceive each category to be typical signs of anxiety or autism-specific. Percentage of responses placing each category into typical or autism-specific were calculated.

\section{Results}

\section{Parent perceptions of anxiety at home, at school, and in the community}

Concurrent validity of parent perceptions of anxiety. Parents reported on whether they thought their child was anxious when at home, at school, and out in the community. As this reflects parental perceptions of anxiety, the first stage was to explore these ratings in terms of their concurrent validity against a standardised measure of anxiety. Firstly, the total score of the ASC-ASD was compared between those 
who stated their child was or was not anxious at home, school, and in the community. Total scores on the ASC-ASD were significantly higher for children who were rated as being anxious at home $(\mathrm{t}(167)=-3.86, \mathrm{p}<.001)$, at school $(\mathrm{t}(159)=-5.98, \mathrm{p}<.001)$, and out in the community $(\mathrm{t}(158)=-4.78, \mathrm{p}<.001)$. Secondly, scores on the ASC-ASD were then explored dependent upon the number of settings in which children were rated as being anxious (0-3). Children who were reported to be anxious across a higher number of settings had a significantly higher total score on the ASC-ASD ( $F(3)=15.1, p<.001$, $\left.\eta p^{2}=.23\right)$. The ASC-ASD subscale scores for children experiencing anxiety in three settings were at least four times the value of scores for children reported to not be experiencing anxiety in any of the settings for all subscales except the Performance Anxiety subscale, where the score of children experiencing anxiety in all three settings was twice that of children reported to not be experiencing anxiety in any of the settings. To summarise, children described as being anxious at home, school, or in the community had higher scores on a parent-completed standardised measure of anxiety than children who were not rated as being anxious in those settings. In addition, as the number of settings a child was reported to be anxious in increased, so did scores on a standardised anxiety measure.

Anxiety at home. As documented in Table 1, a little over a half (52.6\%) of parents stated that they thought their child was anxious at home. There was no significant difference in the proportion of children from the younger and older cohort, nor between males and females, reported by their parents to be anxious at home.

Anxiety at school. As summarised in Table 1,77.6\% of parents stated that they thought their child was anxious at school. There were significantly more children within the older cohort ( $n=71,88.8 \%)$ reported to be anxious at school than in the younger cohort 
$(n=57,67.1 \%)$. The proportion of males reported to be anxious at school did not significantly differ from the proportion of females. 
Table 1.

The Proportion of Parents who Perceived their Child to be Anxious at Home, School, and in the Community for the Total Sample and Split by Cohort and Gender

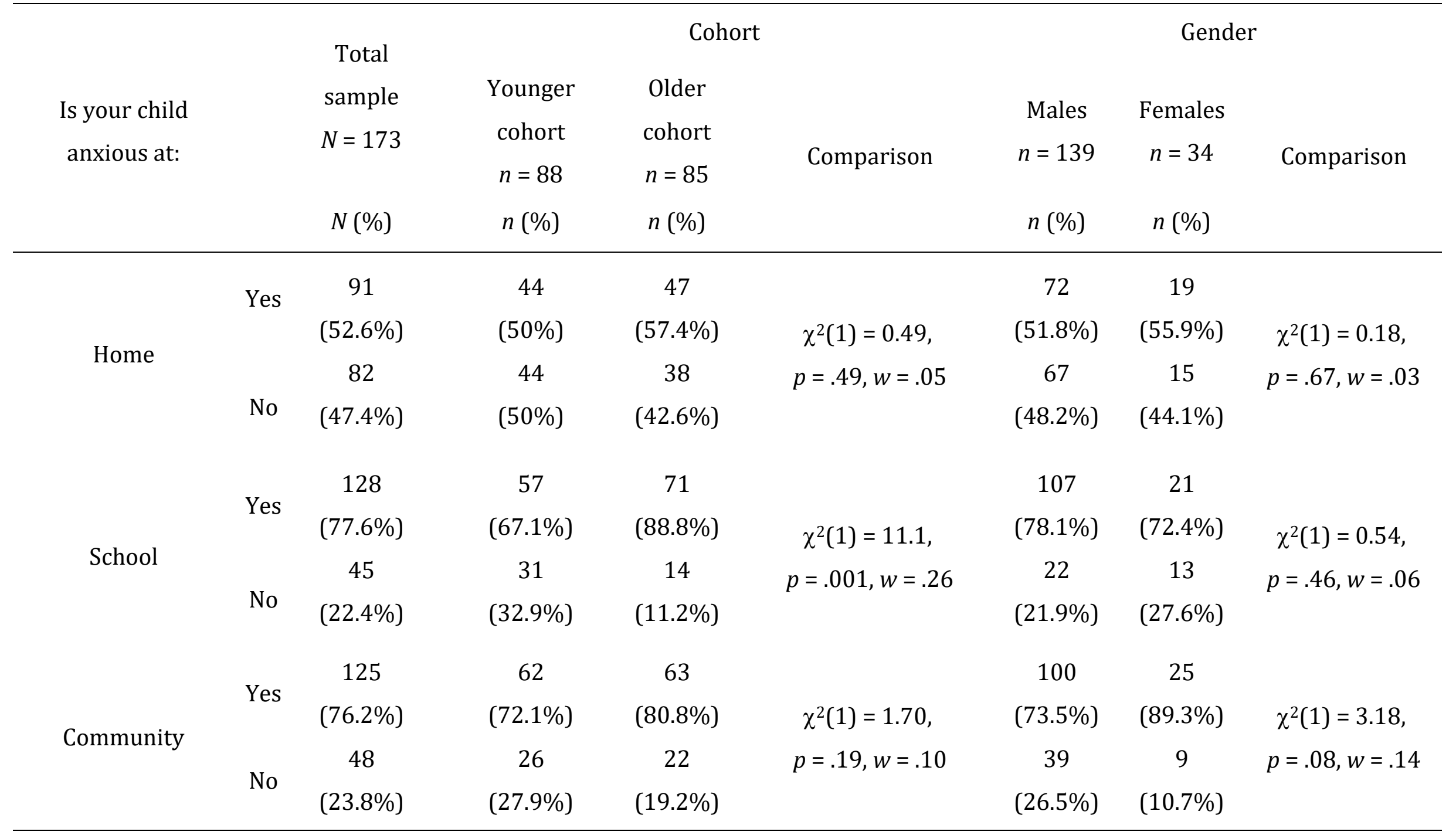


Anxiety in the community. One hundred and twenty five (76.2\%) parents stated that they thought their child was anxious when out in the community. Although there was no significant difference in the proportion of children reported to be anxious in the community between the younger and older cohorts, nor between the genders, both comparisons did have a small effect size (cohort, $w=.10$; gender $w=.14$ ).

Anxiety across multiple settings. Of the 162 parents who provided answers to questions about all three settings, $18(11.1 \%)$ stated that they thought their child was not anxious in any of these settings, 25 (15.4\%) stated their child was anxious in one setting, 50 (30.9\%) in two settings, and 69 (42.6\%) in all three settings.

\section{Parent descriptors of signs of their child's anxiousness}

The content analysis resulted in 40 categories of parent-reported signs of anxiousness across home, school, and community settings. Due to the open-ended nature of the questions, parent responses included those which listed a number of signs from within one or a few categories, to those which listed a number of signs, all of which were placed into different categories. The number of categories (excluding none/unsure $)$ in which parents reported ranged from 1-11 for the home $(\overline{\bar{x}}=3.4, S D=$ 1.7), $1-10$ for school $(\overline{\bar{x}}=2.7, S D=1.6)$, and $1-9(\overline{\bar{x}}=2.8, S D=1.6)$ for the community.

Table 2 list the categories with responses for each category. For ease of presentation, the categories are sorted into five broad areas: changes related to vocalisations; changes in mood, affect, or emotions; changes to a child's movement; physiological changes; and behaviour changes. It is acknowledged that some categories could be associated with multiple areas, so focus should be placed upon the category and description rather than area. The columns on the right hand side of the table show the number of parents who provided at least one description within that category. The 
categories are from highest to lowest frequency of endorsement for signs of anxiousness in the home setting. 
Table 2.

Parent-reported Signs of their Child's Anxiety across Home, School, and Community Settings

\begin{tabular}{|c|c|c|c|c|c|}
\hline Area & Category & Frequently reported example & \multicolumn{3}{|c|}{$\begin{array}{c}\text { Total number (\%) of parents reporting this as } \\
\text { a sign of anxiety }\end{array}$} \\
\hline \multirow{3}{*}{ 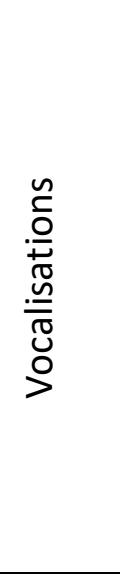 } & $\begin{array}{l}\text { Vocalisations become louder, faster or } \\
\text { shouts }\end{array}$ & Becomes loud, increased volume & $21(23.1 \%)$ & $15(11.7 \%)$ & $18(14.4 \%)$ \\
\hline & $\begin{array}{l}\text { Argues or uses rude/inappropriate } \\
\text { language }\end{array}$ & Swearing, arguments & $6(6.6 \%)$ & $5(3.9 \%)$ & $5(4 \%)$ \\
\hline & $\begin{array}{l}\text { Says that they are anxious, worried or } \\
\text { scared }\end{array}$ & Verbally says "I am worried/anxious" & $4(4.4 \%)$ & $4(3.1 \%)$ & $2(1.6 \%)$ \\
\hline \multirow{7}{*}{ 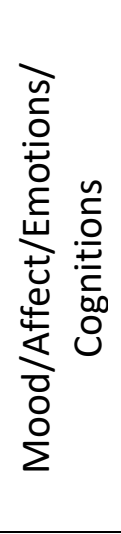 } & Crying or signs of upset & Crying/teary & $20(22 \%)$ & $22(17.2 \%)$ & $18(14.4 \%)$ \\
\hline & Angry or over-reactive & Angry, outbursts & $11(12.1 \%)$ & $20(15.6 \%)$ & $9(7.2 \%)$ \\
\hline & $\begin{array}{l}\text { Difficulties with focus or concentration or } \\
\text { completing mental tasks }\end{array}$ & Unable to focus/think logically & $8(8.8 \%)$ & $11(8.6 \%)$ & $7(5.6 \%)$ \\
\hline & Agitated or restless mood & Agitated & $8(8.8 \%)$ & $6(4.7 \%)$ & $4(3.2 \%)$ \\
\hline & Meltdown & Meltdown & $6(6.6 \%)$ & $6(4.7 \%)$ & $7(5.6 \%)$ \\
\hline & Excitable or silly mood & Laughs at irrelevant things & $3(3.3 \%)$ & $0(0 \%)$ & $1(0.8 \%)$ \\
\hline & Signs of low self-esteem & $\begin{array}{c}\text { Says cannot do task, believes not good } \\
\text { enough }\end{array}$ & $0(0 \%)$ & $9(7 \%)$ & $3(2.4 \%)$ \\
\hline
\end{tabular}




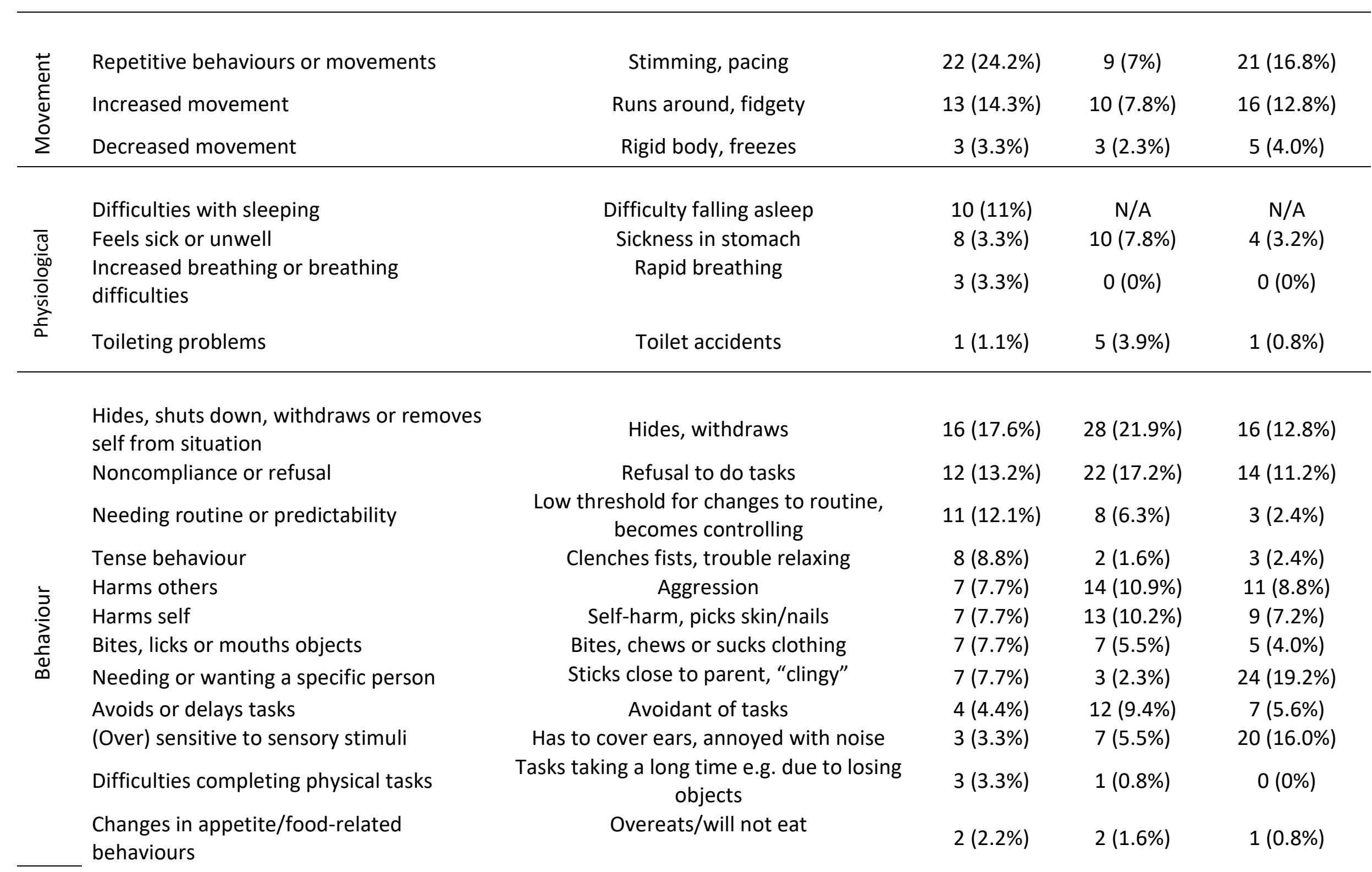


Avoids social interaction

Reluctant or expresses discontent

Runs away

Rushes or impatient behaviour

Wants or asks to go home

Difficulty or refusal to attend school

Difficulties with peer interactions

$\begin{array}{cccc}\text { Does not speak to others, avoids others } & 1(1.1 \%) & 5(3.9 \%) & 16(12.8 \%) \\ \text { Complains, reluctant } & 1(1.1 \%) & 5(3.9 \%) & 6(4.8 \%) \\ \text { Absconds, runs away } & 0(0 \%) & 8(6.3 \%) & 14(11.2 \%) \\ \text { Impatient, rushes } & 0(0 \%) & 1(0.8 \%) & 3(2.4 \%) \\ \text { Asks to go home } & \text { N/A } & 0(0 \%) & 18(14.4 \%) \\ \text { Resists going to school } & \text { N/A } & 7(5.5 \%) & \text { N/A } \\ \begin{array}{c}\text { Misunderstands interactions, begins to get } \\ \text { into arguments with friends }\end{array} & \text { N/A } & 6(4.7 \%) & 0(0 \%)\end{array}$


The data provided in Table 2 highlight the range of signs of anxiousness reported by parents and the differences among the three settings. The most frequently reported signs of anxiousness within the home setting were appearing anxious, worried, or scared (25.3\%), shouting/louder/faster vocalisations (23.1\%), and repetitive behaviours (24.2\%). The most frequently reported signs of anxiousness within the school setting were hiding/shutting down (21.9\%), crying (17.2\%), and noncompliance (17.2\%). The most frequently reported signs of anxiousness in the community were needing/wanting a specific person (19.2\%), repetitive behaviours or movements (16.8\%), and being sensitive to sensory stimuli (16\%). Some behaviours, such as running away, were not reported at home, rarely reported at school (6.3\%), but reported in more than one in 10 children $(11.3 \%)$ who experience anxiety out in the community.

Additional tables that explore parent-reported signs of anxiousness split by gender and by age cohort are provided in the supplementary material. Within these tables it can be noted that some signs of anxiousness may be more prevalent in males than females, for example, vocalisations become louder, faster or shouts is noted in $26.4 \%$ of males at home and $14 \%$ of males at school compared to $10.5 \%$ of females at home and $0 \%$ at school. However, there was also an impact of the environment, suggesting a potential gender by environment interaction, as this behaviour was reported to be present in $15 \%$ of males and $12 \%$ of females when out in the community. Other signs, such as crying and reduced verbal output, were reported in double or triple the proportion of females than in males in some, but not all, of the environments.

\section{Classification of signs of anxiousness as typical or autism-specific. Seven} psychologists and clinical psychologists working and researching in autism and/or anxiety who were blind to the research question (and from outside of the researcher's 
department) independently classified each category (with the frequently reported examples) into either typical or autism-specific signs of anxiousness.

Fifteen of the 40 categories received unanimous ratings. Three of these categories were unanimously rated as autism-specific signs of anxiousness; vocalisations become louder, faster or shouts, repeats or makes repetitive noises, words or phrases and repetitive behaviours or movements. The remaining twelve categories that were all unanimously rated were classified as typical signs of anxiousness; says that they are anxious, worried or scared, appears worried or scared, crying or signs of upset, difficulties with sleeping, feels sick or unwell, increased breathing or breathing difficulties, difficulties with focus or concentrating or completing mental tasks, tense behaviour, changes in appetite/food-related behaviours, reluctant or expresses discontent, wants or asks to go home, and difficulty or refusal to attend school.

A further eight categories received consistent ratings from the majority $(85.7 \%)$ of raters. Four categories were classified as autism-specific signs of anxiousness: excitable or silly mood, harms others, harms self and bites, licks or mouths objects. Four categories were classified as typical signs of anxiousness: reduces verbal output or becomes non-verbal, angry or over-reactive, signs of low self-esteem, increased movement. The remaining 17 categories received mixed ratings.

\section{Parent descriptions of strategies used to reduce anxiousness.}

The content analysis resulted in 42 categories of strategies that parents report using to reduce their child's anxiousness across home, school, and community settings. The number of category strategies (excluding none/unsure) reported by parents ranged from $1-10$ for the home $(\overline{\bar{x}}=2.73, S D=1.5), 1-6$ for school $(\overline{\bar{x}}=2.1, S D=1.2)$, and 1-9 $(\overline{\bar{x}}$ $=2.4, S D=1.6$ ) for the community. 
Table 3 lists the categories with examples, and states the number of parents who reported using at least one strategy within that category. These are sorted into eight broad areas: strategies which are activity based, relaxation based, those relating to communication or identification of the anxiety, those which focus on routine, practice or predictability, those related to sensory issues/needs, those which aim to adjust the task or expectations, those related to nutrition/medication, and those which aim to increase support.

The most frequently reported strategies used within the home setting were talking or explaining (26.4\%), providing/ensuring a calm, safe, quiet location or time alone (26.4\%), and routines or predictability (22\%). The most frequently reported strategies used within the school setting (reported by parents) were parent-teacher collaboration or communication (20.3\%), providing/ensuring a calm, safe, quiet location or time alone (15.6\%), preparing or practising in advance $(13.3 \%)$, and routines or predictability (13.3\%). The most commonly used strategy to reduce anxiousness in the community was preparing or practising in advance (30.4\%), avoiding specific places or removing the trigger for the anxiety (14.4\%), and sensoryrelated strategies $(10.4 \%)$. 
Table 3.

Strategies reported to be helpful in reducing anxiousness in autism across Home, School, and Community Settings

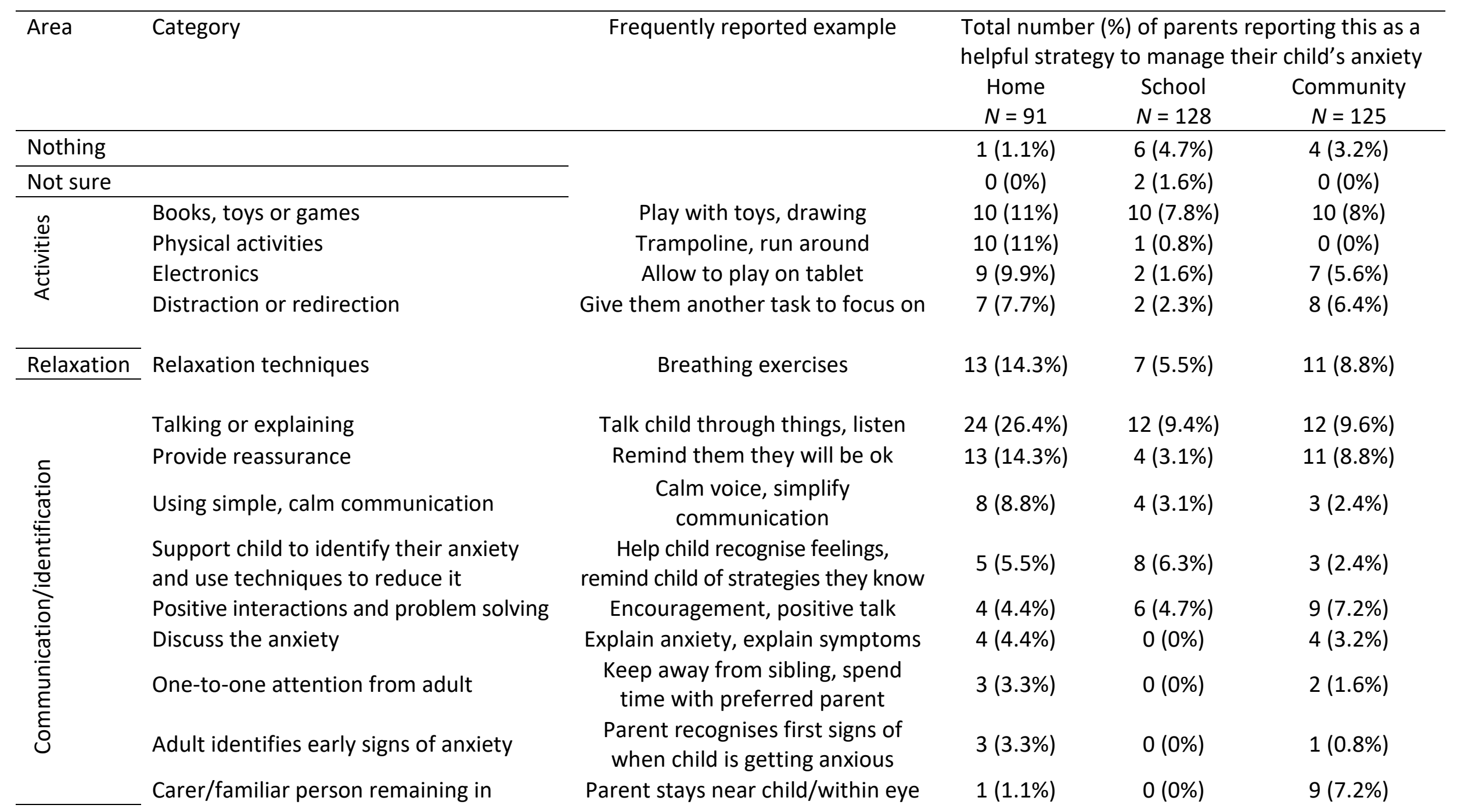


attendance

Educating or informing others
Routines and predictability

Practise and prepare in advance

Visual supports

Set boundaries

Familiarisation

contact

Sharing diagnosis and knowledge of autism

$0(0 \%)$

$0(0 \%)$

$4(3.2 \%)$

Predictable structure, routines

$20(22 \%)$

$17(13.3 \%)$

$12(9.6 \%)$

Do things with child first so they

$$
\text { can do alone }
$$

$15(16.5 \%)$

17 (13.3\%)

$38(30.4 \%)$

Visual schedule, visuals

$11(12.1 \%) \quad 12(9.4 \%)$

$12(9.6 \%)$

Rules, make clear what is expected

$5(5.5 \%)$

$1(0.8 \%)$

$5(4 \%)$

Familiar objects, allow time to get used to surroundings

N/A

$0(0 \%)$

$7(5.6 \%)$

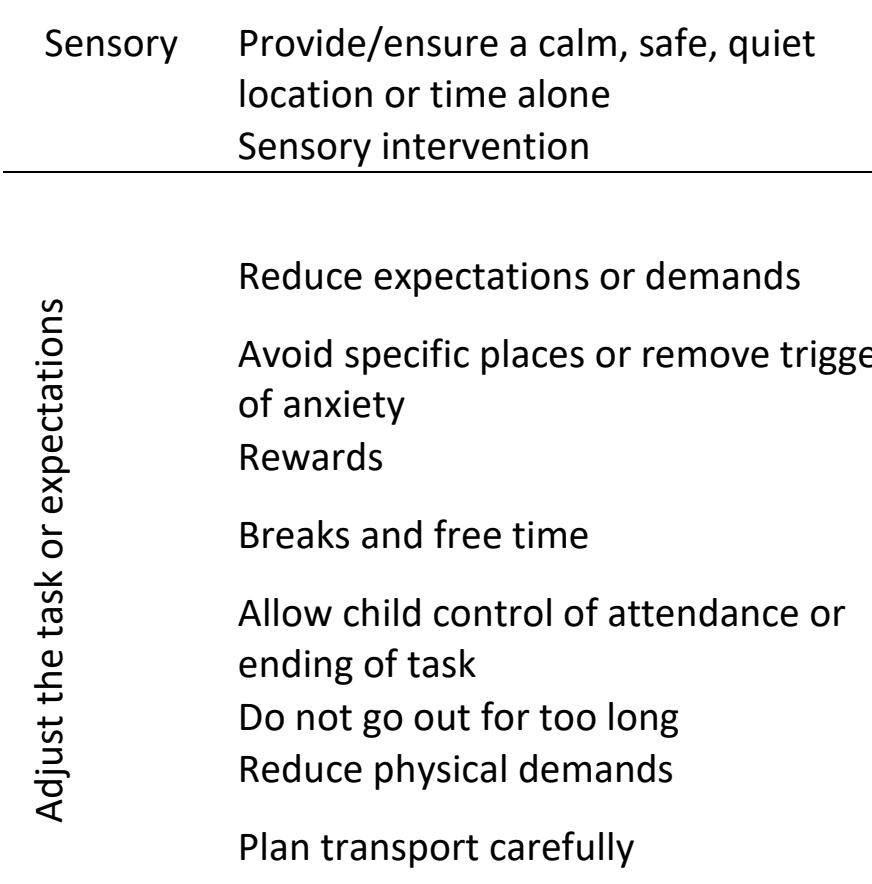

Quite place/room, give space/time to recover/calm down

$24(26.4 \%)$

20 (15.6\%)

$11(8.8 \%)$

Headphones, deep pressure

$9(9.9 \%)$

$11(8.6 \%)$

$13(10.4 \%)$

Reduce pressure, do not ask for too much at once

$8(8.8 \%)$

$8(6.3 \%)$

$12(9.6 \%)$

Remove sources of anxiety, do not take child to certain places

Reward charts

$5(5.5 \%) \quad 4(3.1 \%) \quad 18(14.4 \%)$

Time away from task, take a break to calm down

$2(2.2 \%)$

$2(1.6 \%)$

$6(4.8 \%)$

$2(2.2 \%)$

$6(4.7 \%)$

Do not force child to engage

$0(0 \%)$

$1(0.8 \%)$

$10(8 \%)$

Limit time in community

Use pram

$\mathrm{N} / \mathrm{A}$

N/A

$11(8.8 \%)$

N/A

$\mathrm{N} / \mathrm{A}$

$3(2.4 \%)$

Drive instead of public transport so child can retreat if needed

N/A

$\mathrm{N} / \mathrm{A}$

$2(1.6 \%)$ 


\begin{tabular}{|c|c|c|c|c|c|}
\hline \multirow{2}{*}{ 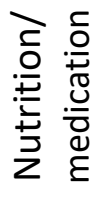 } & Supplements or medication & Medication, probiotics & $4(4.4 \%)$ & $6(4.7 \%)$ & $1(0.8 \%)$ \\
\hline & Access to food or drink & Access to water, chew gum/food & $2(2.2 \%)$ & $4(3.1 \%)$ & $8(6.4 \%)$ \\
\hline \multirow{6}{*}{ 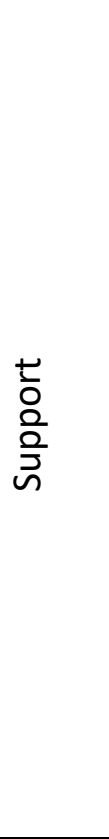 } & Professional input & Psychologist, CBT & $5(5.5 \%)$ & $6(4.7 \%)$ & $1(0.8 \%)$ \\
\hline & $\begin{array}{l}\text { Parent-teacher } \\
\text { collaboration/communication }\end{array}$ & $\begin{array}{l}\text { Parent regularly communicates } \\
\text { with teacher/school }\end{array}$ & $1(1.1 \%)$ & $26(20.3 \%)$ & N/A \\
\hline & $\begin{array}{l}\text { Choosing/being at a school/teacher who } \\
\text { understands child/needs }\end{array}$ & $\begin{array}{l}\text { An understanding teacher, } \\
\text { selecting the right environment }\end{array}$ & $\mathrm{N} / \mathrm{A}$ & $11(8.6 \%)$ & N/A \\
\hline & Withdrawal from school or class & Change schools & $\mathrm{N} / \mathrm{A}$ & $4(3.1 \%)$ & $\mathrm{N} / \mathrm{A}$ \\
\hline & $\begin{array}{l}\text { Learning support and/or people in the } \\
\text { school community }\end{array}$ & $\begin{array}{l}\text { Being involved in school } \\
\text { community, additional learning } \\
\text { support }\end{array}$ & $\mathrm{N} / \mathrm{A}$ & $5(3.9 \%)$ & N/A \\
\hline & $\begin{array}{l}\text { Building a positive child-teacher-parent } \\
\text { relationship }\end{array}$ & Give teacher chocolate & $\mathrm{N} / \mathrm{A}$ & $3(2.3 \%)$ & $\mathrm{N} / \mathrm{A}$ \\
\hline
\end{tabular}


Additional tables that explore the strategies used to reduce anxiousness split by gender and by age cohort are provided in the supplementary material. The strategies used appeared to be relatively consistent between genders. Notable differences were that almost twice as many males $(29.2 \%$ at home, $10 \%$ in the community) find a calm, safe, or quiet location or time alone helpful compared to females $(15.8 \%$ home, $4 \%$ community). In contrast, twice as many parents of females (15.8-16\%) report reducing expectations or demands at home or in the community to be helpful as noted in parents of males $(6.9-8 \%)$. Within the school context, $22.4 \%$ of parents of males on the autism spectrum reported parent-teacher collaboration/communication as an important strategy compared to $9.5 \%$ of parents of females. Three $(14.3 \%)$ parents of females on the autism spectrum reported engaging with learning support and/or the school community compared to $1.9 \%$ of parents of males. Within the younger cohort, visual supports were used in $14.5-15 \%$ of children across the three settings compared to 4.2 $8.5 \%$ of children in the older cohort. Parents within the older cohort reported using books, toys, and games at home and in the community in $12.7-14.9 \%$ of children compared to $3.2-6.8 \%$ of children in the younger cohort.

\section{Discussion}

This study adds to the small but growing literature base reporting on the presentation of anxiety in children on the autism spectrum using qualitative or bottomup methods, and further highlights the importance of recognising behavioural (in addition to emotional and cognitive) aspects of anxiety in children on the autism spectrum. The utilisation of parent-led descriptions of anxiety and anxiousness, in addition to parent responses on standardised assessment items, is important given the ongoing discussion and debate about the possibility of typical and atypical anxiety presentations within individuals on the spectrum (see Kerns \& Kendall, 2012) and 
critical to inform the development of (or adaptations towards) anxiety measures and interventions suitable for an autistic population.

\section{Parent-reported Signs of Anxiety across Settings}

Anxiety is recognised as one of the most common co-occurring diagnoses for children on the autism spectrum (van Steensel, Bogels \& Perrin, 2011), resulting in "an urgency to recognise and treat anxiety in this population" (Vasa, Keefer, Reaven, South, \& White, 2018). The majority (88.9\%) of parents in this study felt that their child was anxious in at least one setting, with almost half of the sample (42.6\%) reporting their child being anxious across all three settings. A little over three quarters of parents felt their child experienced anxiety within the school environment (77.6\%), with more children within the older cohort being reported as experiencing anxiety at school than in the younger cohort (with a medium effect size). The proportion of parents in this study who reported their child as anxious at school and at home also aligned with the subclinical anxiety levels suggested in past research (Strang et al., 2012; Vasa et al., 2013).

The potential for anxiety to present differently across contexts has been relatively unexplored within children on the autism spectrum. There are limited studies reporting on data from multiple informants and to our knowledge, no studies asking a single informant to report on signs of anxiety in multiple settings. Although there is some overlap in the signs noted across environments, there is a difference in both the frequency and range of signs of anxiousness reported by parents across settings. There are also possible gender by environment interactions, although the small sample of females reported to experience anxiousness ( $n=19-25$ across settings) means that these explorations need to be interpreted with caution. Together, these results highlight the importance not only of gathering information on the presentation 
of anxiety from multiple informants, but also of asking a single informant to describe anxiety across multiple settings. This information can enhance our knowledge of anxiousness and anxious symptomatology in children on the autism spectrum and enable us to work towards valid and reliable methods of measurement of anxietyrelated behaviours.

The use of open-ended questions and a modest sample size allowed for a wide variety of responses, not restrained by forced-choice questions existing in traditional questionnaires or checklists. The tendency to report observable behaviours as signs of anxiety affirms the work of Ozsivadijan et al. (2012) and Bearss et al. (2016) who drew their conclusions from focus groups with smaller samples across a broader age range. All the observable signs reported in both of these previous studies were reported by parents in the current study, providing increasing evidence for the importance of the behavioural manifestations of anxiety in children on the spectrum and the presence of atypical signs of anxiety. The coding of the parent-reported signs of anxiousness as typical (i.e. DSM-consistent) or autism-specific suggests that at present, there is greater consistency in interpreting behaviours as typical anxiety symptomatology than autismspecific markers of possible atypical anxiety. This is unsurprising, as the discussion around autism-specific and atypical anxiety is relatively new, and in its infancy both within research and clinical settings. This highlights the need for the combination of further, careful research describing anxiety in individuals with autism, and translation of such research findings into clinical practice.

The challenge of assessing anxiety among children on the autism spectrum is that it can be complex to disentangle autism, anxiety, and other overlapping psychiatric disorders. The limited range of well-validated tools highlights the need for comprehensive approaches to assess anxiety in autism. Bearss et al. (2016) have begun 
work on translating the data from their interview subthemes into a parent-rated anxiety measure for children on the autism spectrum and combining it with 20 items from the Child and Adolescent Symptom Inventory (CASI) Anxiety scale in order to assess both typical and atypical signs of anxiety. Our findings suggest that future research should aim to develop not only further autism-specific assessments which tap both traditional and atypical signs of anxiety, but also ones that aim to incorporate information from multiple informants (e.g., child, parent, teacher) with each informant reporting across different contexts (home, school, community) to form a more holistic assessment and description of the presentation of anxiety. The use of more reliable, valid, and autism-considerate measures of anxiety will assist with intervention planning and the evaluation of intervention strategies within and across contexts.

\section{Parent-reported Strategies to Manage Anxiety}

Parents predominantly used proactive strategies to support the management of their child's anxiety across home, school, and community contexts. These included routines, practice and predictability, sensory support, and adjustments to tasks and expectations. In addition, parents used strategies to aid their child's understanding of the anxiety experience through talking about and explaining the experience and to develop coping strategies such as relaxation techniques. There was limited use of professional support, with only 12 parents utilising these services. This is surprising given that 63 parents reported their child had a clinical diagnosis of anxiety with 27 children currently using anxiety medication. There may have been an underreporting of professional input as parents were asked explicitly about the strategies they employed in different contexts, and they may see professionals as providing broader support across multiple settings, or as providing support for their child's autism, rather than for anxiety specifically. 
There was variability in the strategies which were reported between contexts and although some strategies were frequently reported across the three contexts, this was not simply due to the same parents reporting the use of that strategy across all three settings. For example, 54 parents reported they used "practice and preparing in advance" as a strategy; however, 40 parents used this strategy in only one context, 12 parents used it in two contexts, and two parents applied it across the three contexts. This may suggest that parents are aware that some strategies work better in some contexts than in others, and reported the strategies they viewed as most effective for their child within that context. This context-dependent success of strategies is important clinically, as often clinicians may suggest trialling a strategy in a specific setting (such as the home) before trialling it elsewhere; it may be, however, that that strategy would benefit from being trialled in multiple settings before judging its effectiveness. This also has important implications for trials of interventions for anxiety, highlighting the need to measure anxiety symptomatology and intervention use across multiple settings. Not only can professionals enlighten parents of specific anxiety-reduction techniques, but information from families can in turn inform the development of modifications of anxiety interventions for children on the autism spectrum.

As previously stated, not all children within the community receive a formal diagnosis of anxiety or access an anxiety intervention. Of the current sample, $36.4 \%$ of children were reported by their parents to have a diagnosis of anxiety, but $88.9 \%$ of parents felt that their child was anxious in at least one setting. This suggests that there may be many parents who manage their child's anxiety without a formal anxiety diagnosis and therefore perhaps without professional input, using non-manualised or ad hoc strategies. Many of these strategies were consistent with strategies used by 
parents for managing challenging behaviours in children on the spectrum (O’Nions, Happé, Evers, Boonen, \& Noens, 2018). Other strategies, such as providing reassurance, have been known for many years to maintain and increase anxiety in typically developing children (Rector, Kamkar, Cassin, Ayearst, \& Laposa, 2011). For parents without access to formal interventions, additional support is needed in order for families to effectively address sub-clinical anxiety and to assist parents to effectively manage their child's anxiety using evidence-based techniques.

Unique to the school context was the importance of parent-teacher collaboration or communication (20.3\%) and of having a specific person the child is able to go to for support when needed (10.9\%). Given the high proportion of parents who stated their child was anxious in the school context compared to home and community, and the increase in anxiety at school with age, identifying anxiety and suitable management strategies would appear to be important. Strategies such as having a specific person the child is able to go to for support could be proactively introduced to allow the child to develop a good relationship with that specific person before their anxiety reaches clinical levels. There is limited work exploring the presentation of anxiety in autism at school (see discussion in Adams, Simpson \& Keen, In Press), highlighting the need for further work in this area.

\section{Limitations and Future Directions}

There are several limitations of the present study. The sample was selfselecting and it is unknown whether families who did not choose to enroll differed from families who did choose to enroll in the study. There is therefore a possible ascertainment bias, as participating parents were those who were motivated to take part. While the community diagnosis of autism was confirmed via diagnostic reports together with a score above the cut-off on a widely accepted and validated measure of 
autism characteristics (SCQ), no direct independent diagnostic assessments were conducted. Whilst there was no independent assessment of child anxiety or collection of information on specific anxiety subtypes, the study did not focus upon diagnosed anxiety, instead reporting on parent perceptions of the presence and presentation of anxiety symptomatology and anxiousness in children on the spectrum. Having an assessment of IQ or ability would also have allowed for exploration of signs of anxiety within children with comorbid intellectual disability, an area that is significantly underresearched (Adams \& Oliver, 2011; Flynn et al., 2017).

It is recognised that parents were asked to report on signs of anxiety within the school setting, where they may not have the opportunity to directly observe their child. Parents may have relied on child self-report, teacher discussions or other methods to identify the presentation and strategies in this setting; the source and validity of such information was not explored in this study and may have varied across participants. This highlights the need for a replication of this study with informants who are able to comment on behaviours within this setting, for example, teachers or the children themselves. It also highlights the importance of carefully selecting multiple informants in both research and clinical settings, so that the presentation and impact of anxiousness can be described by people who see the child in each specific setting.

Whilst the online questionnaire enabled the use of a large sample size from a diverse geographical area, this was at the expense of more in-depth probing or clarification that could have been obtained in an interview setting. However, it is possible that parents may have misinterpreted some of the question, or lost focus when answering the question. The sample of expert psychologists and clinical psychologists was small $(n=7)$, but was an international group specialised in anxiety, autism and autism and anxiety. Future research could obtain qualitative data in an interview 
setting utilising the signs and strategies contained within the current article to serve as prompts to ascertain more in-depth information. There is also a need to further explore the possible differences in presentation between males and females, and whether this is consistently different between environments.

\section{Conclusion}

This study combines closed- and open-answer questions to explore the presentation of anxiousness in autism outside of the constraints of symptoms listed on a standardised questionnaire. This is important as, in the words of one of the respondents, "anxiety is a big multi-faceted issue, not a two-minute survey".

The results in this study support those documented in previous qualitative studies with smaller samples, reaffirming how anxiety and anxiousness present behaviourally, rather than solely cognitively or emotionally, in children on the autism spectrum. The finding that anxiety presentations may differ between settings is novel and one that requires further exploration. The documentation of differing strategies being helpful in different settings has important clinical implications and should inform future school- and home-based intervention trials.

\section{References}

Adams, D., \& Oliver, C. (2011). The expression and assessment of emotions and internal states in individuals with severe or profound intellectual disabilities. Clinical Psychology Review, 31, 293-306. doi:10.1016/j.cpr.2011.01.003

Adams, D., Simpson, K. \& Keen, D. (In Press). School-related anxiety symptomatology in a non-clinical sample of primary-school aged on the autism spectrum. Journal of School Psychology 
Baron-Cohen, S., \& Wheelwright, S. (1999). 'Obsessions' in children with autism or Asperger syndrome. Content analysis in terms of core domains of cognition. British Journal of Psychiatry, 175, 484-490.

Bearss, K., Taylor, C. A., Aman, M. G., Whittemore, R., Lecavalier, L., Miller, J., . . . Scahill, L. (2016). Using qualitative methods to guide scale development for anxiety in youth with autism spectrum disorder. Autism, 20, 663-672. doi:10.1177/1362361315601012

Beidel, D. C., Turner, S. M., \& Dancu, C. V. (1985). Physiological, cognitive and behavioral aspects of social anxiety. Behaviour research and therapy, 23, 109-117.

Birmaher, B., Brent, D., Chiapetta, L., Bridge, J., Monga, S., \& Baugher, M. (1999). Psychometric properties of the screen for child anxiety related emotional disorders (SCARED): A replication study. Journal of the American Academy of Child \& Adolescent Psychiatry, 38, 1230-1236.

Chesnut, S. R., Wei, T., Barnard-Brak, L., \& Richman, D. M. (2017). A meta-analysis of the social communication questionnaire: Screening for autism spectrum disorder. Autism, 21, 920-928. doi:10.1177/1362361316660065

den Houting, J., Adams, D., Roberts, J., \& Keen, D. (2018). Exploring anxiety symptomatology in school-aged autistic children using an autism-specific assessment. Research in Autism Spectrum Disorders, 50, 73-82. doi:10.1016/j.rasd.2018.03.005

Dey, I. (1993). Qualitative data analysis: A user friendly guide for social scientists. New York, NY: Taylor \& Francis.

Flynn, S., Vereenooghe, L., Hastings, R. P., Adams, D., Cooper, S. A., Gore, N., . . Waite, J. (2017). Measurement tools for mental health problems and mental well-being in 
people with severe or profound intellectual disabilities: A systematic review. Clinical Psychology Review, 57, 32-44. doi:10.1016/j.cpr.2017.08.006

Johnson, E., \& Hastings, R. P. (2002). Facilitating factors and barriers to the implementation of intensive home-based behavioural intervention for young children with autism. Child: Care, Health and Devolpment, 28, 123-129. doi:10.1046/j.13652214.2002.00251.x

Keen, D., Adams, D., Simpson, K., den Houting, J., \& Roberts, J. (2017, December 5). Anxiety-related symptomatology in young children on the autism spectrum. Autism, Advance online publication, 1-9. doi:10.1177/1362361317734692

Kerns, C., \& Kendall, P. (2012). The presentation and classification of anxiety in autism spectrum disorder. Clinical Psychology: Science and Practice, 19, 323-347. doi:10.1111/cpsp.12009

Kerns, C., Kendall, P., Berry, L., Souders, M. C., Franklin, M. E., Schultz, R. T., . . Herrington, J. (2014). Traditional and atypical presentations of anxiety in youth with autism spectrum disorder. Journal of Autism and Developmental Disorders, 44, 2851-2861. doi:10.1007/s10803-014-2141-7

Lecavalier, L., Gadow, K. D., DeVincent, C. J., \& Edwards, M. C. (2009). Validation of DSM-IV model of psychiatric syndromes in children with autism spectrum disorders. Journal of Autism and Developmental Disorders, 39, 278-289. doi:10.1007/s10803008-0622-2

Lecavalier, L., Wood, J. J., Halladay, A. K., Jones, N. E., Aman, M. G., Cook, E. H., . . Scahill, L. (2014). Measuring anxiety as a treatment endpoint in youth with autism spectrum disorder. Journal of Autism and Developmental Disorders, 44, 1128-1143. doi:10.1007/s10803-013-1974-9 
Magiati, I., Lerh, J. W., Hollocks, M. J., Uljarevic, M., Rodgers, J., McConachie, H., . . Hardan, A. (2017). The measurement properties of the spence children's anxiety scale-parent version in a large international pooled sample of young people with autism spectrum disorder. Autism Research, 10, 1629-1652. doi:10.1016/B978-0-12805122-1.00014-4

Magiati, I., Ozsivadjian, A., \& Kerns, C. (2017). Phenomenology and presentation of anxiety in autism spectrum disorder. In C. M. Kerns, P. Renno, E. Storch, P. C. Kendall, \& J. Wood (Eds.), Anxiety in children and adolescents with autism spectrum disorder: Evidence-based assessment and treatment (pp. 33-54). Oxford, UK: Academic Press. O’Nions, E., Happé, F., Evers, K., Boonen, H., \& Noens, I. (2018). How do parents manage irritability, challenging behaviour, non-compliance and anxiety in children with autism spectrum disorders? A meta-synthesis. Journal of Autism and Developmental Disorders, 48, 1272-1286. doi:10.1007/s10803-017-3361-4

Ozsivadjian, A., Knott, F., \& Magiati, I. (2012). Parent and child perspectives on the nature of anxiety in children and young people with autism spectrum disorders: a focus group study. Autism, 16, 107-121. doi:10.1177/1362361311431703

Polanczyk, G. V., Salum, G., A., Sugaya, L. S., Caye, A., \& Rohde, L. A. (2015). Annual Research Review: A meta-analysis of the worldwide prevalence of mental disorders in children and adolescents. Journal of Child Psychology and Psychiatry, 56, 345365. doi:10.1111/jcpp.12381

Rector, N. A., Kamkar, K., Cassin, S. E., Ayearst, L. E., \& Laposa, J. M. (2011). Assessing excessive reassurance seeking in the anxiety disorders. Journal of Anxiety Disorders, 25, 911-917. doi:10.1016/j.janxdis.2011.05.003

Roberts, J. Adams, D., Heussler, H., Keen, D., Paynter, J., Trembath, D., Westerveld, M. \& Williams, K. (2018). Protocol for a prospective longitudinal study investigating the 
participation and educational trajectories of Australian students with autism. BMJ Open, 8, e017082.

Robertson, A. E., Stanfieid, A. C., Watt, J., Barry, F., Day, M., Cormack, M., \& Melville, C. (2018). The experience and impact of anxiety in autistic adults: A thematic analysis. Research in Autism Spectrum Disorders, 46, 8-18. doi:10.1016/j.rasd.2017.11.006

Rodgers, J., Wigham, S., McConachie, H., Freeston, M., Honey, E., \& Parr, J. R. (2016). Development of the anxiety scale for children with autism spectrum disorder (ASC-ASD). Autism Research, 9, 1205-1215. doi:10.1002/aur.1603

Rutter, M., Bailey, A., Berument, S., Lord, C., \& Pickles, A. (2003). Social communication questionnaire. Los Angeles, CA: Western Psychological Services.

Spain, D., Sin, J., Harwood, L., Mendez, M. A., \& Happé, F. (2017). Cognitive behaviour therapy for social anxiety in autism spectrum disorder: A systematic review. Advances in Autism, 3, 34-46. doi:10.1108/AIA-07-2016-0020

Strang, J. F., Kenworthy, L., Daniolos, P., Case, L., Wills, M. C., Martin, A., \& Wallace, G. L. (2012). Depression and anxiety symptoms in children and adolescents with autism spectrum disorders without intellectual disability. Research in Autism Spectrum Disorders, 6, 406-412. doi:10.1016/j.rasd.2011.06.015

Sze, K. M., \& Wood, J. J. (2008). Enhancing CBT for the treatment of autism spectrum disorders and concurrent anxiety. Behavioural and Cognitive Psychotherapy, 36, 403-409. doi:10.1017/S1352465808004384

Trembath, D., Germano, C., Johanson, G., \& Dissanayake, C. (2012). The experience of anxiety in young adults with autism spectrum disorders. Focus on Autism and Other Developmental Disabilities, 27, 213-224. doi:10.1177/1088357612454916

Ung, D., Selles, R., Small, B. J., \& Storch, E. A. (2015). A systematic review and metaanalysis of cognitive-behavioral therapy for anxiety in youth with high-functioning 
autism spectrum disorders. Child Psychiatry \& Human Development, 46, 533-547. doi:10.1007/s10578-014-0494-y

van Steensel, F., Bögels, S., \& Perrin, S. (2011). Anxiety disorders in children and adolescents with autistic spectrum disorders: A meta-analysis. Clinical Child and Family Psychology Review, 14, 302-317. doi:10.1007/s10567-011-0097-0

Vasa, R. A., Carroll, L. M., Nozzolillo, A. A., Mahajan, R., Mazurek, M. O., Bennett, A. E., ... Bernal, M. P. (2014). A systematic review of treatments for anxiety in youth with autism spectrum disorders. Journal of Autism and Developmental Disorders, 44, 3215-3229. doi:10.1007/s10803-014-2184-9

Vasa, R. A., Kalb, L., Mazurek, M., Kanne, S., Freedman, B., Keefer, A., . . Murray, D. (2013). Age-related differences in the prevalence and correlates of anxiety in youth with autism spectrum disorders. Research in Autism Spectrum Disorders, 7, 13581369. doi:10.1016/j.rasd.2013.07.005

Vasa, R. A., Keefer, A., Reaven, J., South, M., \& White, S. (2018). Priorities for advancing research on youth with autism spectrum disorder and co-occurring anxiety. Journal of Autism and Developmental Disorders, 48, 925-934. doi:10.1007/s10803-0173320-0

Weisbrot, D. M., Gadow, K. D., DeVincent, C. J., \& Pomeroy, J. (2005). The presentation of anxiety in children with pervasive developmental disorders. Journal of Child and Adolescent Psychopharmacology, 15, 477 - 496. doi: 10.1089/cap.2005.15.477

White, S. W., Oswald, D., Ollendick, T., \& Scahill, L. (2009). Anxiety in children and adolescents with autism spectrum disorders. Clinical Psychology Review, 29, 216229. doi:10.1016/j.cpr.2009.01.003 
Running Head: PARENT DESCRIPTORS OF ANXIOUSNESS IN AUTISM 41 